\title{
Combination immunotherapy with Interleukin-2 and CTLA-4 blockade decreases tumor growth and improves overall survival
}

\author{
Joseph R Broucek ${ }^{1 *}$, Tasha Hughes', Erica J Huelsmann², Joseph L Poshepny², Carl E Ruby ${ }^{1,2}$, Andrew Zloza ${ }^{2,3}$, \\ Howard L Kaufman ${ }^{1,2,3}$
}

From Society for Immunotherapy of Cancer 28th Annual Meeting

National Harbor, MD, USA. 8-10 November 2013

\section{Background}

Combination immunotherapy is quickly gaining attention in the field of cancer treatment. IL-2, a cytokine which activates $\mathrm{T}$ cells, and ipilimumab, a monoclonal antibody that blocks CTLA-4, are both approved as monotherapy for metastatic melanoma. To date, combination immunotherapy with IL- 2 and anti-CTLA-4 has not been adequately investigated. We hypothesized that that this combination may work synergistically owing to its distinct but complementary mechanisms of $\mathrm{T}$ cell activation.

\section{Methods}

C57BL/6 mice (10 per group) were challenged with B16-F10 melanoma (via intradermal injection with 120,000 cells) on day 0 . Four groups were treated via intraperitoneal injection: -Group 1 (combination): IL-2 (100,000 units in $100 \mu \mathrm{l})$ every $12 \mathrm{~h}$ on days 4-8 and anti-CTLA-4 $(100 \mu \mathrm{g}$ in $100 \mu \mathrm{l}$ ) on days 3,6 , and 9; -Group 2 (IL-2 only): IL-2 $(100,000$ units in $100 \mu \mathrm{l})$ every $12 \mathrm{~h}$ on days $4-8$ and IgG $(100 \mu \mathrm{g}$ in $100 \mu \mathrm{l}$ ) on days 3,6 , and 9; -Group 3 (antiCTLA-4 only): PBS (100 $\mu \mathrm{l})$ every $12 \mathrm{~h}$ on days $4-8$ and anti-CTLA-4 (100 $\mu \mathrm{g}$ in 100 $\mu \mathrm{l})$ on days 3, 6, and 9; -Group 4 (placebo): PBS $(100 \mu \mathrm{l})$ every $12 \mathrm{~h}$ on days $4-8$ and IgG $(100 \mu \mathrm{g}$ in $100 \mu \mathrm{l})$ on days 3,6 , and 9 . Tumor area (length"width) was measured every other day until death of the animal or until tumors reached $100 \mathrm{~mm} 2$, when animals were sacrificed as per institutional protocols. Primary outcomes included tumor size and overall survival.

\section{Results}

Tumor growth was significantly reduced with combination IL-2 and anti-CTLA-4. Specifically, on day 14, the

${ }^{1}$ Department of Surgery, Rush University Medical Center, Chicago, IL, USA Full list of author information is available at the end of the article mean tumor area with combination IL-2 and anti-CTLA4 was $2 \mathrm{~mm} 2$, while in the anti-CTLA- 4 only, IL-2 only, and placebo groups it was 14,29 , and $68 \mathrm{~mm} 2$, respectively ( $\mathrm{P}<0.01$ for all comparisons, except IL- 2 only versus anti-CTLA-4 only [not significant]). At day 30 , the overall survival with combination IL-2 and anti-CTLA-4 was $50 \%$, while with IL- 2 only and anti-CTLA- 4 only it was $10 \%$ and $20 \%$, respectively $(\mathrm{P}<0.01$ for all comparisons). All animals treated in the placebo group succumbed to their tumors by day 19 . These findings were confirmed in a second experiment with similar results.

\section{Conclusions}

Combination immunotherapy with IL-2 activation and CTLA-4 blockade significantly decreases tumor growth and increases overall survival when compared to IL-2 or anti-CTLA-4 monotherapy or placebo. The role of CD8+ effector versus $\mathrm{CD} 4+$ regulatory $\mathrm{T}$ cells in the success of this immunotherapy is ongoing and will be included in our formal presentation. Ultimately, we aim to translate this work into a combination immunotherapy clinical trial.

\begin{abstract}
Authors' details
'Department of Surgery, Rush University Medical Center, Chicago, IL, USA. 2Department of Immunology/Microbiology, Rush University Medical Center, Chicago, IL, USA. ${ }^{3}$ Department of Internal Medicine, Rush University Medical Center, Chicago, IL, USA.
\end{abstract}

Published: 7 November 2013

doi:10.1186/2051-1426-1-S1-P70

Cite this article as: Broucek et al: Combination immunotherapy with Interleukin-2 and CTLA-4 blockade decreases tumor growth and improves overall survival. Journal for ImmunoTherapy of Cancer 20131 (Suppl 1):P70. 